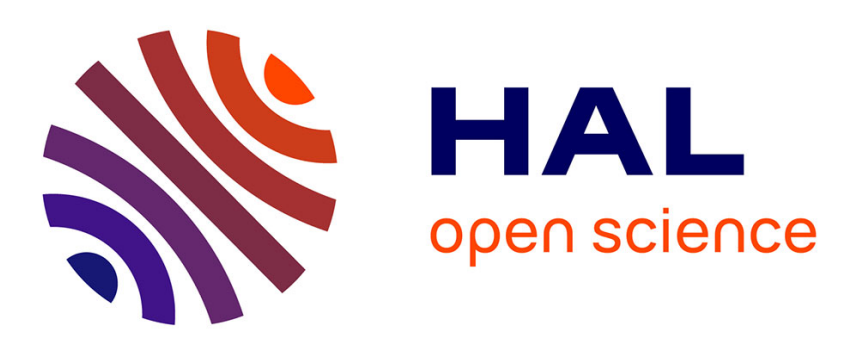

\title{
In Situ Real-Time Quantitative and Qualitative Monitoring of Molecular Contamination
}

Emilie Vanhove, Thomas Tondu, Jean-Marc Roussel, Delphine Faye, Pascale Guigue

\section{- To cite this version:}

Emilie Vanhove, Thomas Tondu, Jean-Marc Roussel, Delphine Faye, Pascale Guigue. In Situ RealTime Quantitative and Qualitative Monitoring of Molecular Contamination. Journal of Spacecraft and Rockets, 2017, 53 (6), p. 1166-1171. 10.2514/1.A33505 . hal-01488099

\section{HAL Id: hal-01488099 \\ https://hal.science/hal-01488099}

Submitted on 13 Mar 2017

HAL is a multi-disciplinary open access archive for the deposit and dissemination of scientific research documents, whether they are published or not. The documents may come from teaching and research institutions in France or abroad, or from public or private research centers.
L'archive ouverte pluridisciplinaire HAL, est destinée au dépôt et à la diffusion de documents scientifiques de niveau recherche, publiés ou non, émanant des établissements d'enseignement et de recherche français ou étrangers, des laboratoires publics ou privés. 


\title{
In Situ Real-Time Quantitative and Qualitative Monitoring of Molecular Contamination
}

\author{
E. Vanhove, $\stackrel{*}{-}$ T. Tondu, $\stackrel{ \pm}{ \pm}$ and J. F. Roussel $\stackrel{ \pm}{ }$ \\ ONERA - The French Aerospace Lab, 31055 Toulouse, France \\ and \\ Delphine Faye $\stackrel{\S}{\text { and }}$ Pascale Guigue $\mathbb{I}$ \\ Centre National d'Etudes Spatiales, 31401 Toulouse, France
}

\section{DOI: $10.2514 / 1 . \mathrm{A} 33505$}

\begin{abstract}
The quantitative and qualitative monitoring of contamination is an important issue for the mitigation of the risks induced by contamination. In situ and real-time measurements of contamination levels are currently performed with good accuracy by using quartz microbalances. However, they have to be completed by chemical analyses to identify the nature of the contaminants. Unfortunately, in situ techniques are limited to a very rough characterization. A transfer of the samples is then required, which prohibits real-time monitoring and may lead to the partial degradation of the samples. To tackle this challenge, an experimental technique, coupling thermogravimetric analysis and mass spectrometry, has been developed at ONERA. This method takes advantage of a preseparation of the species through the thermogravimetric analysis. A numerical tool was moreover developed to process automatically experimental thermogravimetric analysis/mass spectrometry data. It enables determination of the contribution of each species (deconvolution of thermogravimetric analysis measurements species by species, both for quartz crystal microbalance measurements and mass spectrometry spectra) and extraction of the mass spectrum of each of these species. The results of this analysis are presented for the characterization of an adhesive widely used for space applications. The deconvolution in species matches very well the experimental data.
\end{abstract}

\section{Nomenclature}

$\begin{array}{lll}\mathrm{d} m(t) / \mathrm{d} t & = & \text { total mass reemission rate in } 10^{-8} \mathrm{~g} \cdot \mathrm{cm}^{-2} \cdot \mathrm{s}^{-1} \\ M_{j}(t) & = & \text { mass spectrometry measurement } \\ & & \text { for mass-to-charge ratio } j \text { arbitrary unit } \\ m_{i}(t) & \text { dimensionless mass reemitted for chemical } \\ m / z & = & \text { species } i \\ m_{0 i} & \text { mass-to-charge ratio in } u \\ & \text { initial mass of the chemical species } i \\ s_{j i} & \text { in } 10^{-8} \mathrm{~g} \cdot \mathrm{cm}^{-2}\end{array}$

\section{Introduction}

$\mathbf{M}$ OLECULAR contamination can seriously compromise the objectives of a mission. It can indeed significantly degrade the performance of measuring instruments and solar panels, as well as thermal control systems. To mitigate this risk, a number of tests are systematically performed on ground during the phases of design, manufacture, and validation of the satellite [1,2].

Received 5 November 2015; revision received 30 May 2016; accepted for publication 9 June 2016; published online 15 September 2016. Copyright $(\odot)$ 2016 by the American Institute of Aeronautics and Astronautics, Inc. All rights reserved. Copies of this paper may be made for personal and internal use, on condition that the copier pay the per-copy fee to the Copyright Clearance Center (CCC). All requests for copying and permission to reprint should be submitted to CCC at www.copyright.com; employ the ISSN 0022 4650 (print) or 1533-6794 (online) to initiate your request.

*Research Engineer, Space Environment Department; emilie.vanhove@ onera.fr.

${ }^{\dagger}$ Research Engineer, Space Environment Department, ONERA 2, Avenue Edouard Belin.

${ }^{\ddagger}$ Head of Space Environment Department, Space Environment Department; Jean-Francois.Roussel@onera.fr.

${ }^{\S}$ Material and Contamination Engineer, Quality Insurance; delphine.faye @ cnes.fr.

${ }^{\top}$ Material and Contamination Engineer, Quality Insurance; pascale. guigue@cnes.fr.
The most widely used test to characterize this contamination consists of monitoring the mass of deposited contaminants per unit area by using quartz microbalances [ $\underline{3}-\underline{5}]$. This measurement is both very reliable and very sensitive $[\underline{6}, \overline{7}]$. However, this technique does not allow determination of the chemical nature of each of the contaminants nor its relative proportion in the deposit. To complement this measurement, various chemical analyses can thus be carried out, such as in situ mass spectrometry, gas chromatography coupled to mass spectrometry, Fourier transform

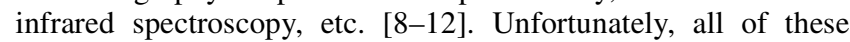
techniques have severe limitations.

To begin with, a large part of them in fact require a transfer of samples. This transfer is undesirable for two reasons. First, a transfer is highly likely to irremediably deteriorate samples (pressure or temperature changes, spontaneous evolution of samples over time such as reemission or phase change, etc.). This would raise doubts extremely hard to clear up, for example, the extent to which the quartz crystal microbalance (QCM) measurements can really be compared with the results of the chemical analysis.

The second reason is that this transfer prevents the chemical characterization from being performed in real time. The time evolution information is thus definitively lost. This information is, however, of particular interest. On the one hand, it allows a much better correlation between the quantitative measurements (the deposited mass) and the chemical analyses (nature of contaminants). On the other hand, it enables the kinetic study of contamination phenomena (outgassing, transport, reemission, etc.).

Some of the most recent physical models are indeed based on the individual behavior of each chemical species of a mixture of contaminants $[4,13]$. The same is true for models of contaminationUV synergy $[\underline{14}, \underline{15}]$. As of today, however, common experimental measurements only monitor the total contaminant deposit. In the future, a real-time analysis able to quantitatively separate the contributions of each species would, on the other hand, thus enable 1) a much better assessment of contamination mechanisms at work, 2) a much more realistic extraction of model parameters and a much better characterization of the kinetic properties of each contaminant, and 3) a more relevant and comprehensive analysis of tests performed on ground.

Some techniques, such as Fourier transform infrared spectroscopy and mass spectrometry (MS) can, however, be operated in situ. 
Unfortunately, it can be pretty hard to implement them with the required sensitivity and, above all, to interpret the measurements, because they result from a complex mixture of a priori unknown contaminants. As a consequence, these analyses are generally not quantitative and are often limited to a very rough characterization.

To meet this challenge, ONERA has developed an analytical technique for the real-time and in situ qualitative (nature of each deposited contaminant) and quantitative (deposited mass of each species) monitoring of molecular contamination by coupling the thermogravimetric analysis (TGA) and MS analyses [16]. In the first part of this study, the experimental setup, the measurements, and a preliminary analysis of these data are presented. The method is applied to the characterization of a real contaminant, the ScotchWeld EC-2216, an adhesive frequently used in the aerospace industry. Although TGA results in a kind of rough species separation, this separation is incomplete. As a result, the mass spectrum measured is still the one of an unknown mixture of contaminants. Because such spectra are very complex, the analysis of experimental data as shown in the first part requires a great deal of expertise and is unreasonably time consuming. Furthermore, it does not allow the extraction of the mass spectra of each one of the chemical species. ONERA therefore upgraded its numerical tool COMPEX to enable the automatic analysis of the TGA/MS experimental data. The second part briefly describes this tool and presents the results of this digital processing.

\section{Coupling of TGA and MS Analyses}

In this first part, the experimental setup, the protocol used, the experimental data, and their analysis are explained.

\section{A. Experimental Setup}

Contamination photons synergy is the experimental facility dedicated to the study of molecular contamination at ONERA. An effusion cell $\left(20-200^{\circ} \mathrm{C}\right)$ generates a controlled molecular beam of contaminants toward a collection plate (steel plate of typically $10 \mathrm{~cm}^{2}$ ) and a QCM (Maxtek $6 \mathrm{MHz}$ ), both individually temperature controlled from -100 to $100^{\circ} \mathrm{C}$ (cooling with liquid nitrogen and resistance heating, system developed by ONERA). During the TGA, contaminants reemitted from the collection plate are analyzed using a mass spectrometer (Bruker). QCM frequencies are sensitive to temperature. This dependence has been characterized and subtracted to TGA measurements.

\section{B. Experimental Protocol}

The protocol used was specifically designed for the characterization of real contaminants. It includes four steps of outgassing. At the end of each step, a TGA/MS measurement is performed.

The four outgassing steps were performed at 75, 100, 125, and $150^{\circ} \mathrm{C}$. Each step lasted about $20 \mathrm{~h}$. The contaminants were collected on the collection plate at $-80^{\circ} \mathrm{C}$. The temperature profiles of the effusion cell and of the plate are given in Fig. 1. A TGA was performed at the end of each step at the heating rate of $2^{\circ} \mathrm{C} / \mathrm{min}$. Reemitted contaminants were then analyzed by mass spectrometry. The TGA/MS data recorded after the first outgassing step at $75^{\circ} \mathrm{C}$ were not exploited because the amount of deposited contaminant was too low.

\section{Experimental Data}

ATGA/MS experiment consists of one TGA and 250 mass signals. For each $\mathrm{m} / \mathrm{z}$ between 1 and 250 , the intensity is measured as a function of time, and thus as a function of temperature, because the experiment carried out is a TGA.

Figure 2 shows the three TGAs monitored after the outgassing steps at 100,125 , and $150^{\circ} \mathrm{C}$ (i.e., total mass reemission rate from the QCM). By way of illustration, the measurements obtained for the mass 47 for the three outgassing steps are given in Fig. 3 .

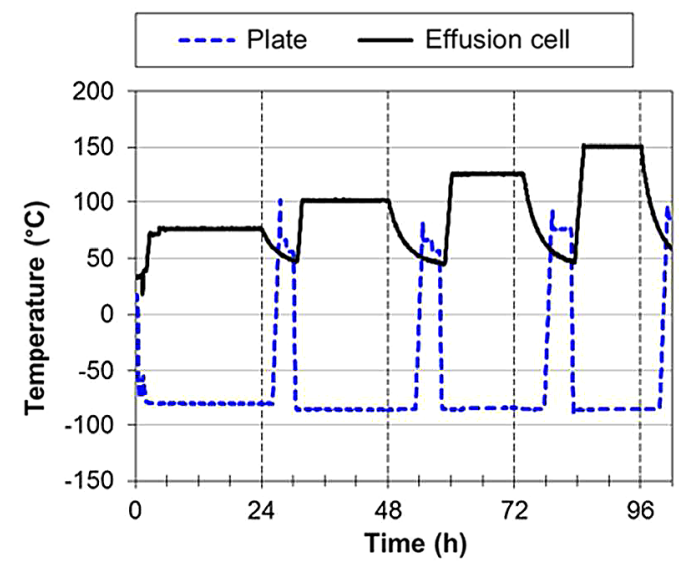

Fig. 1 Temperatures of effusion cell and collection plate.

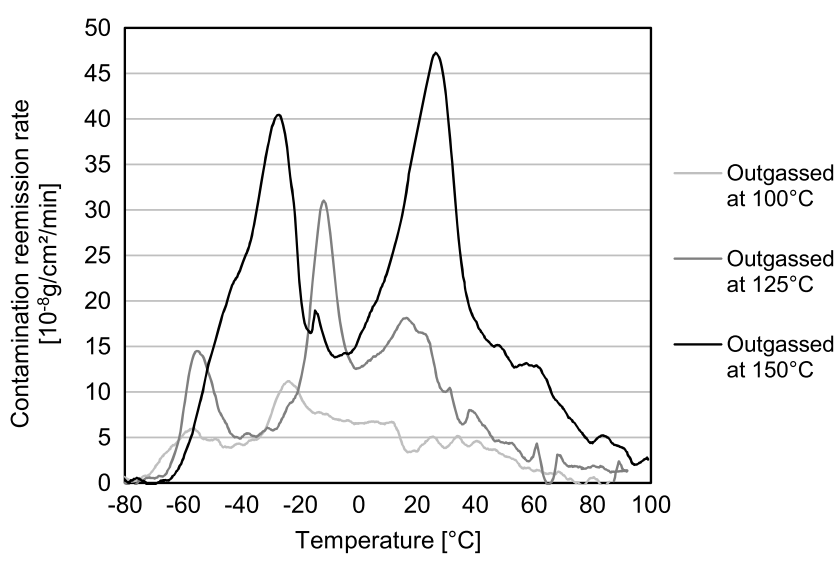

Fig. 2 TGA measurements, outgassing steps at 100,125 , and $150^{\circ} \mathrm{C}$.

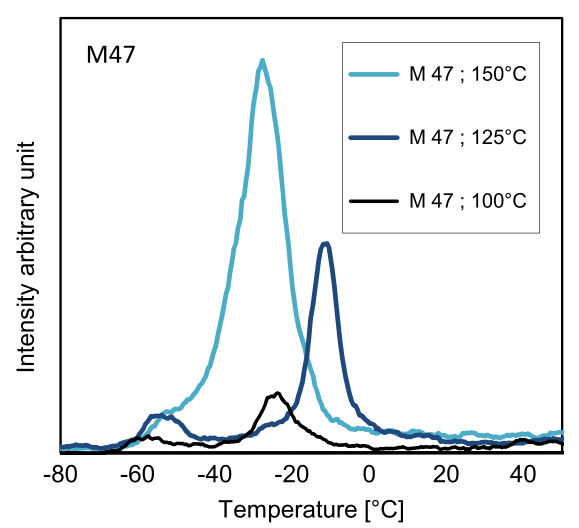

Fig. 3 TGAs performed after the outgassing steps at 100,125 and $150^{\circ}$ $\mathrm{C}, \mathrm{m} / \mathbf{z} 47$.

\section{Experimental Data Analysis: Deconvolution 1}

Although TGA performs some species separation, it remains incomplete. Furthermore, peak emissions partly overlap. The issue of the deconvolution is therefore to use mass spectrometry data to separate the contributions of the different chemical species and to identify them (Fig. 4). However, several species contribute to each mass signal monitored by mass spectroscopy, making the interpretation of experimental data quite difficult.

As a consequence, the deconvolution was performed by identifying the masses that stem from one single species. Such an identification was based on the following criteria:

1) A mass signal having a unique and very narrow peak (e.g., mass 47, Fig. 3 ) most likely corresponds to a single species. 

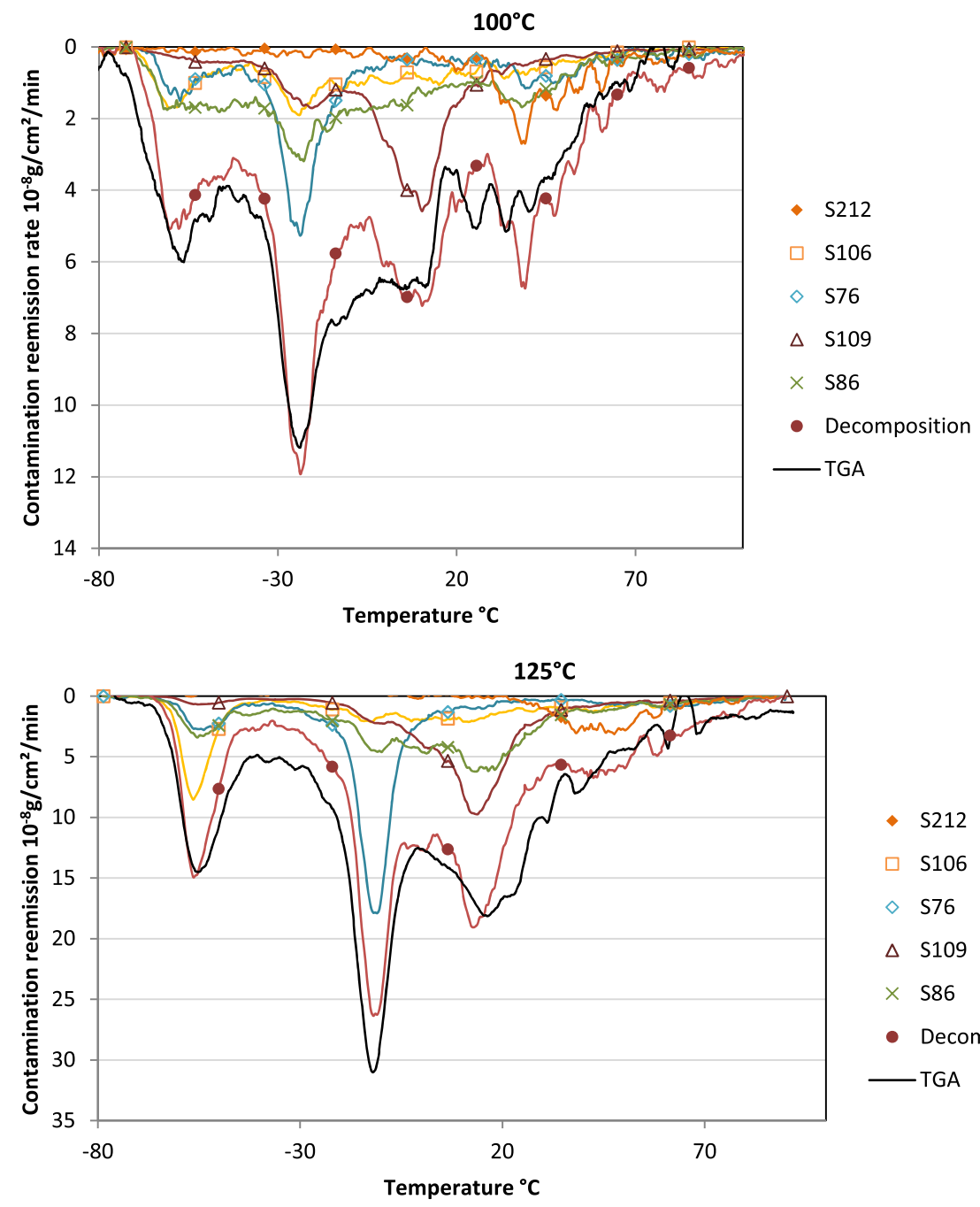

$\diamond \mathrm{S} 76$

$\triangle S 109$

$\times \quad \mathrm{S} 86$

- Decomposition

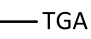

$150^{\circ} \mathrm{C}$

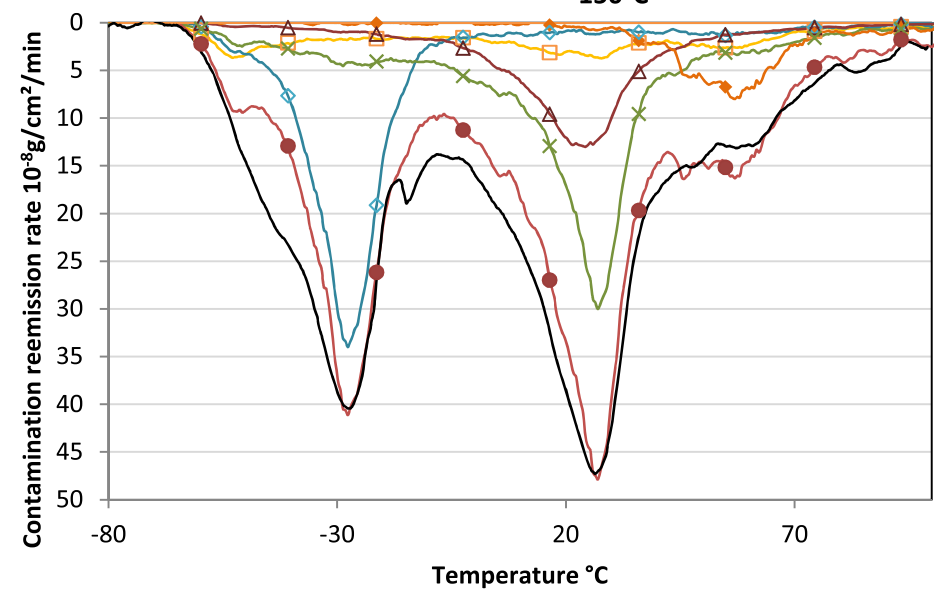

- S212

$\square$ S106

$\diamond \mathrm{S} 76$

$\triangle S 109$

$\times S 86$

- Decomposition

TGA

Fig. 4 Species-by-species deconvolutions of TGA measurements, outgassing steps at 100,125 , and $150^{\circ} \mathrm{C}$.

2) When masses separated by at least several unities have identical complex signals (several peak emissions), they are very likely to be emitted from a single species. By contrast, isotopes and, in particular, carbon-13 lead to $M+1$ and $M+2$ masses that have identical signals but may well correspond to several species.

3) The heaviest masses have a lower probability to correspond to several species.

4) Finally, the temperatures that correspond to a peak or a shoulder of the TGA performed after the step at $150^{\circ} \mathrm{C}$ were identified. The analysis thus focused on the masses that show a sharp peak for these temperatures.
These masses were called characteristic masses and were used to identify the species. The TGA, measured by the QCM, is the total mass reemission rate. It can be described as the sum of the masses of each species. It is therefore a linear combination of the characteristic masses of these species. It was thus finally possible to reproduce the experimental TGAs with only five main contaminants, for which the characteristic masses were the masses 106, 76, 109, 86, and 212 (Fig. 4). The mass 76 saturates for the outgassing step at $150^{\circ} \mathrm{C}$. However, as the masses 47 and 76 were proportional for all the outgassing steps (Fig. 5), the mass 47 was used instead of the mass 76 for the TGA deconvolution. A very satisfying fit of experimental data 


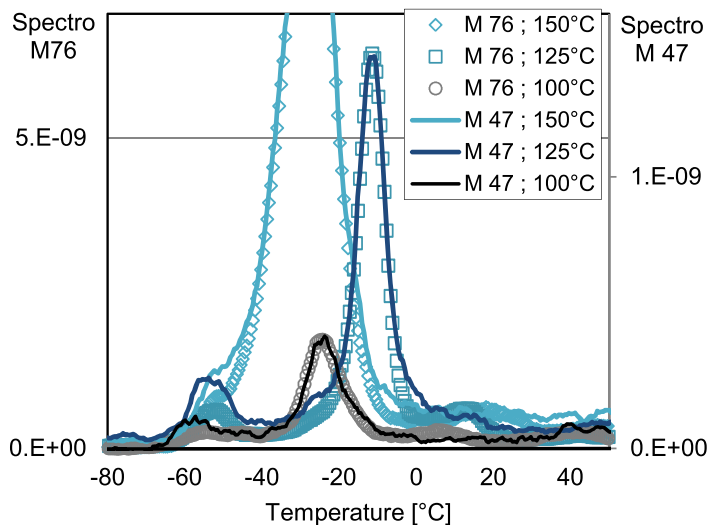

Fig. 5 TGAs performed after the outgassing steps at 100,125 and $150^{\circ}$ C, $m / \mathrm{z} 47$ and 76 .

was thereby obtained for the three outgassing temperatures, although the reemission profiles recorded after each temperature step differ widely. In this first analysis, the less abundant contaminants were deliberately neglected. Thus, for instance, for the outgassing step at $150^{\circ} \mathrm{C}$, a contaminant, emitted at approximatively $-45^{\circ} \mathrm{C}$, seems to be lacking.

The first part of this paper described how to determine in situ and in real time the contribution of each species of a contaminant mixture during a TGA experience by coupling the TGA and MS analyses. This TGA deconvolution enables for each identified species a first estimation of its reemission properties as well as of its total deposited mass at the end of each outgassing step. Based on this deconvolution, thanks to the masses identified as characteristic of a single species, it is also possible to determine the contribution of each species to the deposit during the outgassing step. This technique thus provides new information that could be exploited to validate contamination models (outgassing and reemission) and to extract their parameters. However, this method has two limitations: First, the interpretation of experimental data is very tedious, and second, it is not possible to extract in this way the mass spectrum of each species to identify them. This is why the numerical tool COMPEX developed by ONERA was enhanced to enable the automatic processing of TGA/MS data.

\section{Numerical Processing of TGA/MS Data}

\section{A. Numerical Model}

The available experimental data are the TGA measurements, on the one hand, and the mass spectrometry data, on the other hand. A TGA (total mass reemission rate) can be described as the sum of the contributions of each species $i$.

$$
\operatorname{TGA}(t)=\frac{\mathrm{d} m(t)}{\mathrm{d} t}=\sum_{i} m_{0 i} \frac{\mathrm{d} m_{i}(t)}{\mathrm{d} t}
$$

In the same way, we can break down the mass spectrometry measurement $M_{j}(t)$ monitored for each mass $j$ :

$$
M_{j}(t)=\sum_{i} s_{j i} m_{0 i} \frac{\mathrm{d} m_{i}(t)}{\mathrm{d} t}
$$

The unknowns are then the initial mass and the mass spectrum $s_{j i}$ of each species.

A higher weight ( $w>1$, here 50) is, moreover, given to the TGA. The error function is thus

$$
\begin{aligned}
& w\left[\sum_{i} m_{0 i} \frac{\mathrm{d} m_{i}(t)}{\mathrm{d} t}-\operatorname{TGA}(t)\right]^{2} \\
& +\sum_{j}\left[\sum_{i} s_{j i} m_{0 i} \frac{\mathrm{d} m_{i}(t)}{\mathrm{d} t}-M_{j}(t)\right]^{2}
\end{aligned}
$$

At each step, the optimum spectra $s_{j i}$ opt are computed using the least-squares method, the masses being considered fixed. Current spectra $s_{j i}(n)$ are replaced at the step $n+1$ by spectra defined by Eq. (ㅁ):
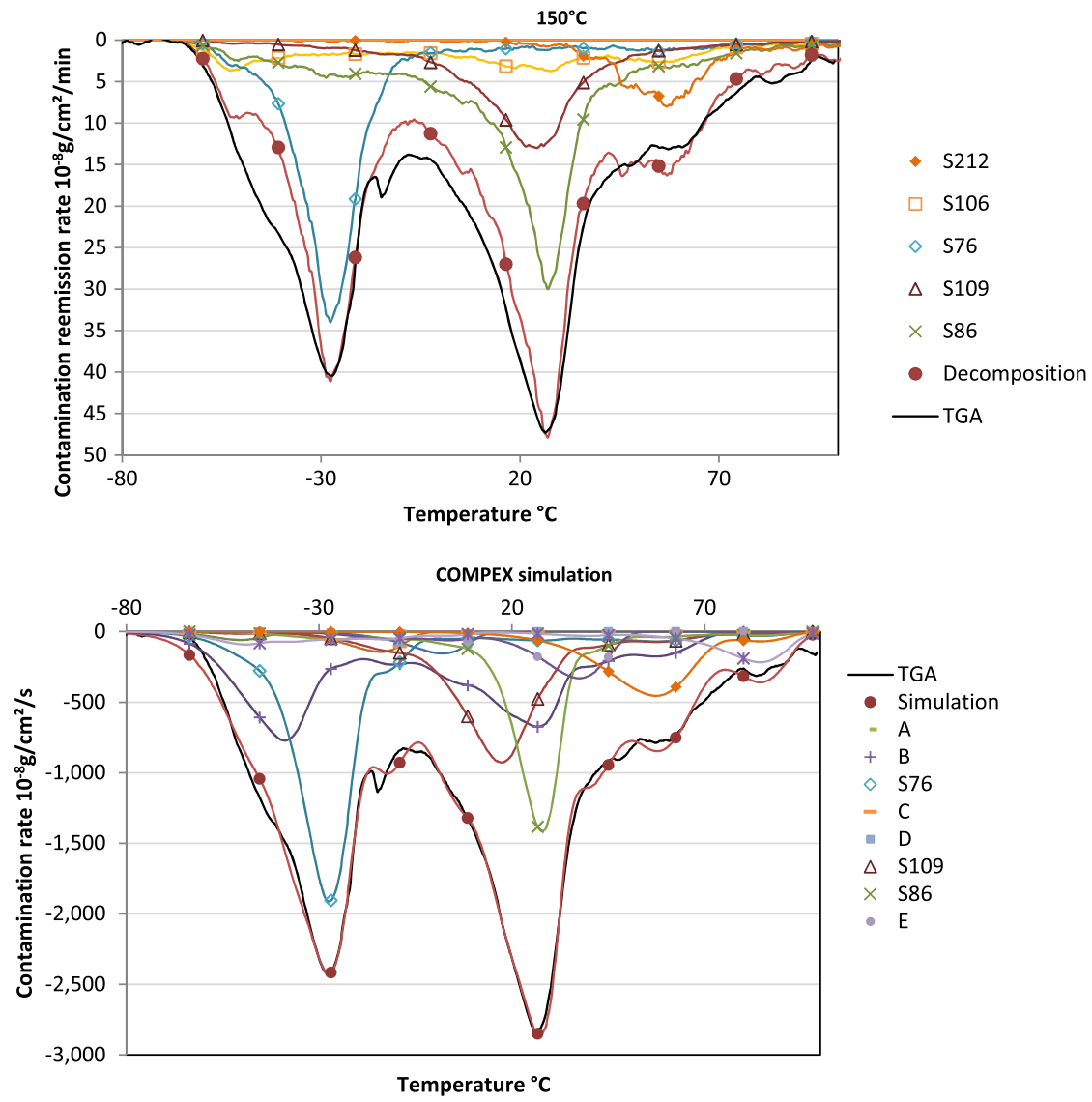

Fig. 6 Species-by-species deconvolution of TGA measurements: (top) deconvolution 1, (bottom) numerical simulation. 


$$
s_{j i}(n+1)=\frac{s_{j i}(n)+s_{j i} \mathrm{opt}}{2}
$$

Equation (2) being linear in $s_{j i}$, the error decreases. The spectra are then fixed and the masses are treated by the same method. Because the error is reduced with each iteration, the algorithm converges and a local minimum is found.

\section{B. Simulation: Deconvolution 2}

The TGA performed immediately after the outgassing step at $150^{\circ}$ $\mathrm{C}$ is simulated. Ten species are used for this simulation.

To evaluate the ability of our numerical tool to process TGA/MS data, we compare the results of the experimental data analysis described in the first part of this paper (deconvolution 1) with those provided by the numerical tool (deconvolution 2). In particular, it is important to assess whether or not the chemical species showing up in the two deconvolutions are identical. To this end, we use the masses (masses monitored by mass spectrometry) that had been identified as characteristic of a chemical species. These are the masses resulting from the contribution of a single species, or at least for which one species is predominant. Thus, for the sake of simplicity, because no other information is at first available, the chemical species are named after their characteristics masses: For instance, the species which is the (quasi) exclusive contributor to the mass 76 (Fig. 3) is called S76.

\section{TGA}

Figure 6 shows, at the bottom, the COMPEX simulation (deconvolution 2) of the TGA performed after the outgassing step at $150^{\circ} \mathrm{C}$ and, at the top, the deconvolution 1 of this TGA. Overall, these two deconvolutions are very similar, which is a very satisfying result. The simulation perfectly fits the experimental TGA. The weights of each species (masses initially deposited, S76, S86, S109, and S212) are very close to the ones of deconvolution 1 . The main differences are slightly different reemission parameters for the species S109 (remission occurs at a lower temperature in the simulation) and the appearance of some additional species (A, B, C, D, E, F), which globally correspond to missing masses in deconvolution 1 .

Indeed, although deconvolution 1 is robust (three outgassing temperatures were properly described with a small number of species) and, as a consequence, reliable, this deconvolution relies on a number of approximations (modest contributions are neglected, etc.). As a result, deconvolution 1 is not an absolute reference but rather a good indication of the likelihood of the results.

\section{Characteristic Masses}

The masses that had been identified as characteristic of a single (or at least predominant) species were the masses 47 (signals of mass 47 and 76 are quasi identical), 109, 86, and 212. These masses are shown in Fig. 7. Again, a really good fit of experimental data is obtained for these characteristic masses (Fig. 7) as well as for all of the monitored mass signals (not shown).

The masses 47 and 212 result almost exclusively from the contributions of the species S76 and S212, respectively, and the species S86 and S109 predominantly contribute to the masses 86 and 109, respectively. The species S76, S212, S86, and S109 were thus properly identified. The contributions of the species S86 and S109 are a bit difficult to distinguish from each other because they have close reemission parameters and mass spectra.

\section{Mass Spectra}

The advantage of the automatic data processing is that it now allows extraction of the mass spectrum of each species. The proper identification of these chemical species would, however, require further data treatment, including the comparison of the spectra with a database and or gas chromatography (GC)/MS data. Nevertheless, typical fragment patterns can yet be observed. For example, in the spectrum of the species $\mathrm{S} 109$ (Fig. ). $\mathrm{C}_{n} \mathrm{H}_{2 n-3}$ fragments clearly appear, which could point at $\mathrm{S} 109$ being an alkyne.
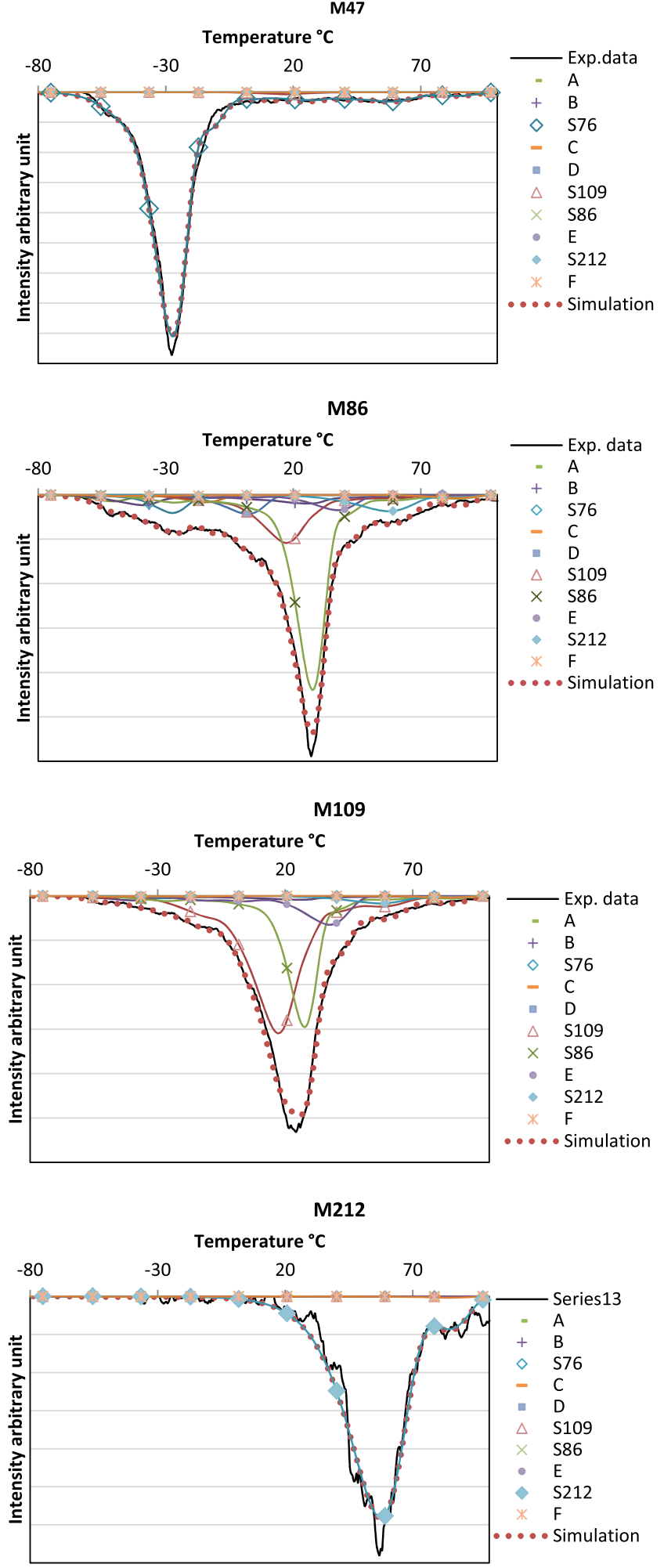

Fig. 7 Species-by-species deconvolution of $m / z$ 47, 86, 109, and 212 .

\section{Conclusions}

The results of this simulation are very promising: They are selfconsistent and in line with the ones of the previous deconvolution. The main species of the mixture of contaminants are properly identified. Further data processing, including the comparison of the spectra with a database, is, however, required to more precisely determine their chemical nature. It would, moreover, be very interesting to compare these spectra with the GC/MS analysis of the EC-2216. 


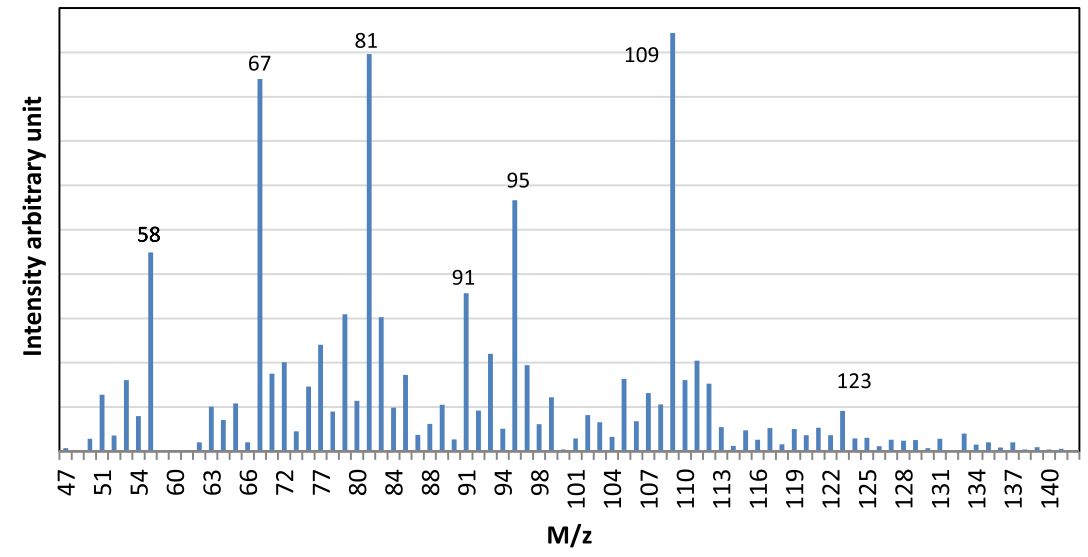

Fig. 8 Mass spectrum resulting from the simulation, deconvolution 2, species S109.

Processing a complete data set (i.e., comprising several TGA performed at different outgassing temperatures) would as well enable a further validation and a more accurate evaluation of the results (initial masses, reemission properties, and mass spectra).

\section{Conclusions}

This paper presented the implementation of the coupling of the TGA and MS analyses and the treatment of the experimental data thus generated. This in situ and real-time characterization method allows the analysis of complex mixtures of contaminants with the objective of determining the nature, quantity, and outgassing and reemission properties of each chemical species of the deposit. Analyzing TGA/ MS experimental data is, however, fastidious and unreasonably time consuming. To overcome this difficulty, a numerical tool able to automatically process such data was developed. This tool enables the species-by-species deconvolution of a TGA experiment and the extraction of the mass spectrum of each identified species. The estimation of reemission properties is also greatly facilitated. Ultimately, the determination of these mass spectra would also allow the deconvolution of the contaminant emitted at each step species by species (characterization of the outgassing properties of each species). The method has been applied to the characterization of an adhesive widely used in space, the Scotch-Weld EC-2216. The results are very promising because the deconvolutions obtained are globally consistent and the simulation fits very well experimental data (TGA and MS data). Further data processing, including the comparison of the spectra with a database, is, however, required to more precisely determine the chemical nature of the species.

\section{Acknowledgments}

The results obtained at ONERA were funded by Centre National d'Etudes Spatiales Research and Technology and ESA Technology Research Programmes, for which the ONERA authors are very grateful.

\section{References}

[1] Tribble, A. C., Fundamentals of Contamination Control, SPIE Press, Bellingham, WA, 2000, pp. 7-60.

[2] "Standard Practice for Preparation of Aerospace Contamination Control Plans," American Soc. for Testing and Materials International, ASTM E1548-09, West Conshohocken, PA, 2009.

[3] "Standard Test Method for Total Mass Loss and Collected Volatile Condensable Materials from Outgassing in a Vacuum Environment," American Soc. for Testing and Materials International, ASTM E595-15, West Conshohocken, PA, 2015.
[4] "Kinetic Outgassing of Materials for Space," ESA Requirements and Standards Div., ECSS-Q-TM-70-52A, Noordwijk, The Netherlands, 2009.

[5] "Standard Test Method for Contamination Outgassing Characteristics of Spacecraft Materials," American Soc. for Testing and Materials International, ASTM E1559-09, West Conshohocken, PA, 2009.

[6] Vashist, S. K., and Vashist, P., "Recent Advances in Quartz Crystal Microbalance-Based Sensors," Journal of Sensors, Vol. 2011, Sept. 2011, Paper e571405.

[7] Wallace, D. A., and Wallace, S. A., "New Generation of Miniaturized, High-Mass-Sensitivity Quartz Crystal Microbalances (QCMs) for Space Applications," Proceedings of SPIE 2864, Optical System Contamination V, and Stray Light and System Optimization, SPIE Press, Bellingham, WA, 1996, pp. 56-71.

[8] Anderson, M. S., "Mass Spectrometry of Spacecraft Contamination Using Direct Analysis in Real-Time Ion Source," Journal of Spacecraft and Rockets, Vol. 51, No. 1, 2014, pp. 374-378. doi:10.2514/1.A32613

[9] Mandrile, L., Giovannozzi, A. M., Pennecchi, F., Saverino, A., Lobascio, C., and Rossi, A. M., "Direct Detection and Quantification of Molecular Surface Contaminants by Infrared and Raman Spectroscopy," Analytical Methods, Vol. 7, No. 6, 2015, pp. 2813-2821. doi:10.1039/C4AY02850C

[10] Pang, H. W., and Zhou, C. L., "Real-Time Molecular Contamination Monitoring During Spacecraft Development," Advances in the Astronautical Sciences, Vol. 117, 2004, pp. 939-944.

[11] "Detection of Organic Contamination of Surfaces by Infrared Spectroscopy," ESA Requirements and Standards Div., ECSS-Q-ST70-05C, Noordwijk, The Netherlands, 2009.

[12] Guyt, J. M., Van Eesbeek, M., and Van Papendrecht, G., "Detection of Organic Contamination on Surfaces by Infrared Spectroscopy," Proceedings of the Photo-Optical Instrumentation Engineers (SPIE), SPIE Press, Bellingham, WA, 2002, pp. 262-271.

[13] Roussel, J.-F., Tondu, T., Paulmier, T., Faye, D., Van Eesbeek, M., and Rampini, R., "Progress on the Physical Approach to Molecular Contamination Modeling," Journal of Spacecraft and Rockets, Vol. 48, No. 2, April 2011, pp. 246-255. doi: $10.2514 / 1.49490$

[14] Tondu, T., Roussel, J.-F., and Faye, D., "New Mechanism for VUV Fixation of Contamination," 12th International Symposium on Materials in a Space Environment - ISMSE12, ESA Communications, Noordwijk, The Netherlands, Sept. 2012.

[15] Roussel, J.-F., Vanhove, E., Tondu, T., Faye, D., and Guigue, P., "Ultraviolet Fixation of Molecular Contamination: Physical Model, Numerical, Implementation and Validation," Journal of Spacecraft and Rockets (to be published). doi: $10.2514 / 1 . A 33504$

[16] Tondu, T., and Roussel, J.-F., "Mixture Effects in Contaminant Reemission," 12th International Symposium on Materials in the Space Environment, ESA Communications, Noordwijk, The Netherlands, 2012.

K. Timothy

Associate Editor 